\title{
A Systematic Review on Cornea Epithelial-Stromal Homeostasis
}

\author{
Ho Lam Wong a Stephanie Hiu Ling Poon ${ }^{a}$ Yashan Bu ${ }^{a}$ Amy Cheuk Yin Lo ${ }^{a}$ \\ Vishal Jhanji $^{\text {b Yau Kei Chan }}{ }^{\text {a Kendrick Co Shih }}{ }^{a}$ \\ a Department of Ophthalmology, Li Ka Shing Faculty of Medicine, University of Hong Kong, \\ Hong Kong, Hong Kong SAR; ' Department of Ophthalmology, University of Pittsburgh Medical Centre, \\ Pittsburgh, PA, USA
}

\section{Keywords}

Cornea $\cdot$ Epithelium · Fibroblasts · Wound healing $\cdot$ Fibrosis

\begin{abstract}
Introduction: This review aims to summarise the role of different cells, genes, proteins and lipid in regulating cornea epithelial-stromal homeostasis. Methods: We performed an Entrez PubMed literature search using keywords "human," "cornea," "epithelial," "stromal," "homeostasis," "fibrosis response," and "pathogenesis" on 24th of September 2019, resulting in 35 papers, of which 18 were chosen after filtering for "English language" and "published within 10 years" as well as curation for relevance by the authors. Results: The 18 selected papers showed that corneal epithelial cells, fibroblasts and telocytes, together with genes such as Klf4, Pax6 and Id found in the cells, play important roles in achieving homeostasis to maintain corneal integrity and transparency. Proteins classified as pro-fibrotic ligands and anti-fibrotic ligands are responsible for regulating cornea stromal fibrosis and extracellular matrix deposition, thus regulators of scar formation during wound healing. Anti-inflammatory ligands and wound repairing ligands are critical in eliciting protec-
\end{abstract}

karger@karger.com www.karger.com/ore

(C) 2020 S. Karger AG, Base

Karger" tive inflammation and promoting epithelial healing, respectively. Protein receptors located on cellular membrane play a role in maintaining intercellular connections as well as corneal hydration. Discussion/Conclusion: These studies prompt development of novel therapeutic strategies such as tear drops or ointments that target certain proteins to maintain corneal homeostasis. However, more in vitro and in vivo studies are required to prove the effectiveness of exogenous administration of molecules in improving healing outcome. Hence, future investigations of the molecular pathways highlighted in this review will reveal novel therapeutic tools such as gene or cell therapy to treat corneal diseases.

(c) 2020 S. Karger AG, Basel

\section{Introduction}

The cornea, comprised of 5 layers, namely the epithelium, Bowman's layer, stroma, Descemet's membrane, and endothelium, is essential for clear vision. It helps refract two-thirds of light onto the retina through the pupil and lens. Also, the cornea acts as a physical barrier to protect internal ocular structures from external insults such 


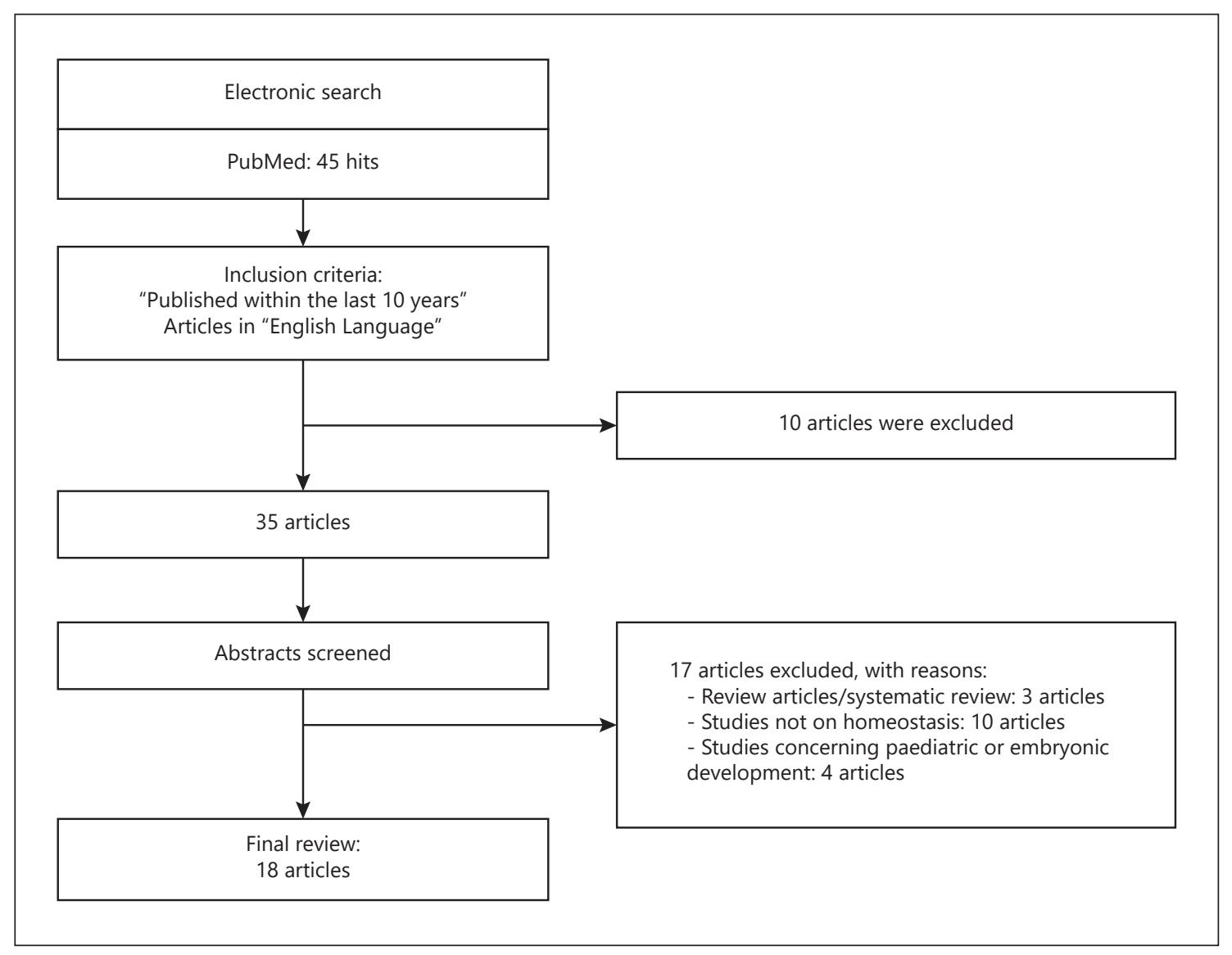

Fig. 1. PRISMA flowchart of search strategy.

as microbial, physical and chemical damage [1]. Given its superficial location, the cornea is externally exposed to the environment and hence prone to various diseases such as keratitis [2], keratopathy [3] and epithelial-stromal scarring [4]. These pathologies will hinder the transparency and smooth curvature of the cornea, which is maintained by keratocytes and collagen extracellular matrix (ECM) in the stroma [5], leading to a loss of refractive power and even ocular morbidity.

Since disrupted epithelial integrity and stromal opacification inhibit clear and focused vision, our cornea has its own homeostatic system to restore tissue transparency after wounding. In face of external disturbances, the cornea will initiate various mechanisms to develop protective inflammation and regulate cell migration and proliferation [6]. Epithelial cells will be continuously replenished by limbal stem cells to maintain an effective physical barrier [7]. Moreover, the cornea has its own dynamic process to repair injured cells through cell signalling cascades to prevent chronic inflammation and infection [8].
Hence, with the regulated immune response to injury, the cornea itself can have an improved healing outcome with minimal tissue changes [9]. Nevertheless, irreversible changes of the cornea such as corneal haze and loss of epithelial basement membrane may occur upon damage. This prompts studies to understand the molecular changes in the cornea to seek novel therapies to restore its homeostasis.

In this study, a systematic review of literature published within these 10 years was performed. This review focused on the pathways of how the cornea itself responds to external changes and maintains its constant condition. The responses of cornea upon scarring to permit protective inflammation were also discussed. We highlighted the cells, genes, proteins and lipid involved in the epithelial and stromal layer that help restore the physiological status of the cornea. Potential therapeutic proteins or cells that can cure or alleviate corneal disorders were also discussed. 
Table 1. Cells involved in cornea epithelial-stromal homeostasis

\begin{tabular}{llll}
\hline $\begin{array}{l}\text { First author [Ref.], } \\
\text { year }\end{array}$ & Cells & Mechanism of action & Effects on corneal homeostasis \\
\hline $\begin{array}{l}\text { Kobayashi [12], } \\
2015\end{array}$ & $\begin{array}{l}\text { Epithelial cells, } \\
\text { fibroblasts }\end{array}$ & $\begin{array}{l}\text { Along with epithelial cells, fibroblasts } \\
\text { upregulate the expression of TGF- } \alpha \text { but } \\
\text { downregulate TGF- } \beta \text { expression in fibroblasts }\end{array}$ & $\begin{array}{l}\text { Interaction between cells promotes } \\
\text { epithelial cells proliferation, migration, } \\
\text { and stratification }\end{array}$ \\
\hline $\begin{array}{l}\text { Marini [77], } \\
2017\end{array}$ & Telocytes & $\begin{array}{l}\text { Partake in intercellular signalling by releasing } \\
\text { extracellular vesicles to communicate with } \\
\text { other cell types }\end{array}$ & $\begin{array}{l}\text { Participate in corneal regeneration and } \\
\text { repair; maintain correct stromal assembly } \\
\text { for corneal transparency }\end{array}$ \\
\hline
\end{tabular}

Table 2. Genes involved in cornea epithelial-stromal homeostasis

\begin{tabular}{llll}
\hline $\begin{array}{l}\text { First author [Ref.], } \\
\text { year }\end{array}$ & Genes & Mechanism of action & Effects on corneal homeostasis \\
\hline $\begin{array}{l}\text { Delp [19], } \\
2015\end{array}$ & Klf4 & $\begin{array}{l}\text { Prevents degradation of epithelial membrane by } \\
\text { suppressing MMP9 secretion; maintains epithelial } \\
\text { layer integrity by expressing tight junctions and } \\
\text { laminin-332 }\end{array}$ & $\begin{array}{l}\text { Maintains the integrity of corneal epithelium } \\
\text { by regulating the formation of basement } \\
\text { membrane and expression cell cycle regulators }\end{array}$ \\
$\begin{array}{llll}\text { Mort [23], } \\
2011\end{array}$ & Pax6 & $\begin{array}{l}\text { Regulates the normal morphology of epithelial, } \\
\text { stromal and endothelial cells }\end{array}$ & $\begin{array}{l}\text { Maintains cell adhesion, movement, normal } \\
\text { mosaic patterns, promoting corneal integrity }\end{array}$ \\
$\begin{array}{l}\text { Mohan [25], } \\
2016\end{array}$ & Id & $\begin{array}{l}\text { Differentially regulated by TGF- } \beta 1 \text { and BMP7 in a } \\
\text { time-dependent manner }\end{array}$ & $\begin{array}{l}\text { Minimizes corneal fibrosis and differentiation } \\
\text { during wound healing }\end{array}$ \\
\hline
\end{tabular}

\section{Methods}

An Entrez PubMed search was conducted on the 24th of September 2019 using the search terms "human," "cornea," "epithelial," "stromal," "homeostasis," "fibrosis response," and "pathogenesis." Only articles published within 10 years and written in English language were included to ensure updated articles are reported. With these criteria, 35 articles were identified. Only in vitro, ex vivo and in vivo set-ups describing the mechanisms of the players or their antagonists involved in the homeostasis of the corneal epithelium and stroma were included. For example, papers concerning the pathways of epithelial-stromal injury and healing or process of stromal keratocytes differentiation were considered as relevant. Furthermore, only original articles were included, while review papers and meta-analyses were excluded. The resulting articles were then manually curated for subject relevance via abstract or full text by H.L.W. and Y.K.C. independently, followed by a discussion on the relevance of identified papers. K.C.S. made the arbitration when both H.L.W. and Y.K.C. disagreed on paper selection, as well as confirmed the articles to be investigated in this study. H.L.W. and Y.K.C. independently scored the selected papers for risk of biases, which were scored with "Low," "Moderate," or "High" for (1) whether a valid model was used to disrupt the cornea homeostasis, (2) sample randomisation, (3) validate measurement, (4) blinded assessment of outcome and (5) appropriate statistical analysis, based on the Critical Appraisal Skills Programme (CASP). A study that satisfied all 5,
3-4, or < 3 criteria was regarded as "Low," "Moderate," and "High" respectively. The relevant papers were stratified based on the cells, genes, proteins and lipid involved in the epithelial-stromal homeostasis. Figure 1 describes the selection process for identified studies.

\section{Results}

In total, 18 in vitro, ex vivo and in vivo studies were reviewed after manual curation (Fig. 1). Five papers were considered as having a low level of bias, such as the use of transgenic animals to evaluate the role of gene, while 13 were regarded as "moderate," including studies blocking certain signalling pathways. For analysis, we grouped our discussion of the finalised papers into the following headings: (1) cells (Table 1), (2) genes (Table 2), (3) proteins (Table 3 ) and (4) lipid (Table 4).

\section{Cell Types}

Corneal epithelial cells (CECs), fibroblasts and telocytes were found to contribute to cornea homeostasis. 
Table 3. Proteins involved in cornea epithelial-stromal homeostasis

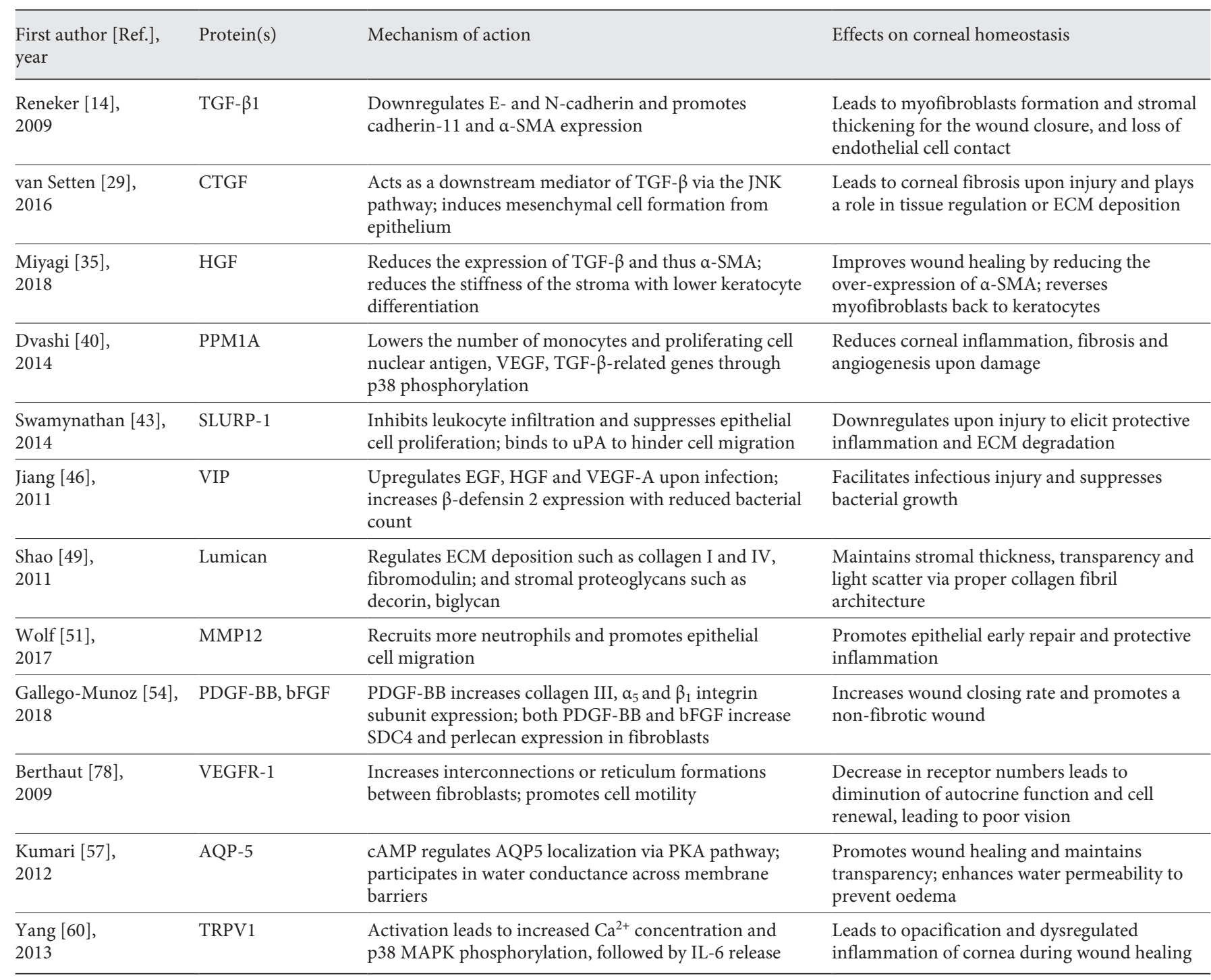

Table 4. Lipid involved in cornea epithelial-stromal homeostasis

\begin{tabular}{llll}
\hline $\begin{array}{l}\text { First author } \\
\text { [Ref.], year }\end{array}$ & Lipid & Mechanism of action & Effects on corneal homeostasis \\
\hline $\begin{array}{l}\text { Rizvi [64], } \\
2011\end{array}$ & Ceramide & $\begin{array}{l}\text { Upregulates HRK expression and JNK phosphorylation, } \\
\text { leading to mitochondrial dysfunction in fibroblasts }\end{array}$ & $\begin{array}{l}\text { Leads to corneal fibroblast cell death and } \\
\text { promotes wound healing }\end{array}$ \\
\hline
\end{tabular}

\section{CECs and Fibroblasts}

Soluble cytokines secreted by fibroblasts were proven to regulate the proliferation of epithelial cells, while growth factors secreted by epithelial cells were shown to affect corneal integrity [10]. In fact, the close interaction between human CECs (HCECs) and human corneal fibroblasts (HCFs) is crucial to corneal homeostasis and development [11]. Culturing epithelial cells on fibroblast-collagen mixture, 9 growth factors, namely fibroblast growth factor 7 , hepatocyte growth factor (HGF), nerve growth factor, epi- 
dermal growth factor (EGF), sarcoma growth factor, transforming growth factor- $\alpha$ (TGF- $\alpha$ ), TGF- $\beta 1, \beta 2$ and $\beta 3$ expression level in HCFs were tested [12]. Among them, only the expression level of TGF- $\alpha$ was increased significantly in HCFs co-cultured with CECs, with an increased level ranging from 12 to 152 times compared to those without CECs. Since TGF- $a$ was found to promote epithelial cell proliferation and migration, fibroblasts were suggested to play a role in influencing epithelial wound healing [13]. Also, stratification of the epithelial layer was only observed when epithelial cells were seeded on HCF-collagen gel, hinting that TGF- $\alpha$ may be essential to maintain a mature corneal epithelial stratification.

Conversely, TGF- $\beta$, especially TGF- $\beta 1,2$ and 3 , mRNA expression was lower in HCFs co-cultured with CECs than that in HCFs alone. Since TGF- $\beta$ was proven to inhibit CEC proliferation, its downregulation may induce minimal inhibition on cell growth and thus improve healing of the epithelium [14]. Thus, CECs interact with HCFs to maintain corneal homeostasis upon injury.

\section{Telocytes}

Telocytes, interstitial cells found in the stromal compartment in different organs, have been characterised with a small cell body with long telopodes, as well as podomeres and podoms [15]. This cell type was believed to partake in intercellular signalling by releasing extracellular vesicles such as exosomes and ectosomes to communicate with other cell types [16]. Telocytes have been newly found in the corneal stroma to maintain a correct ECM organisation. In a normal cornea, cells were found stained positive with markers of telocytes, CD34 and platelet-derived growth factor receptor- $\alpha$. Having long and thin cellular processes, $\mathrm{CD} 34^{+} / \mathrm{c}-\mathrm{kit}^{+}$or $\mathrm{CD} 34^{+} / \mathrm{c}-\mathrm{kit}^{-}$ telocytes, were found in subepithelial or deeper stroma, illustrating that different telocytes subpopulations are densely populated in the stroma. However, in keratoconic cornea, CD $34^{+}$and c-kit ${ }^{+}$stromal cells were decreased and telocytes were distributed in an uneven manner. A significant drop in telocytes was found compared to normal ones. Furthermore, telocytes from keratoconic cornea showed abnormal morphology such as loss of organelles, darkened cytoplasm and shrinkage of telopodes. Telocytes were suggested to play a critical role in maintaining a highly organised collagen matrix, thus contributing to high corneal transparency and stability. In a keratoconic cornea, the loss of telocytes, in particular the c$\mathrm{kit}^{+}$subtype, leads to altered ECM arrangement and ultimately adversely affect the regeneration process, leading to the bulging of cornea into a cone-like shape.

\section{Genes}

Krüppel-like factor-4 (Klf4), Pax6 and Inhibitor of differentiation (Id) gene were indicated in maintaining the epithelial-stromal homeostasis.

\section{Klf4 Gene}

Well-regulated tissue turnover and basal membrane integrity are crucial to the homeostasis of corneal epithelium [17]. Klf4 gene is a highly expressed transcription factor in the cornea and was found to play an integral role in the mature of mouse ocular surface [18]. It has been shown to play a role in regulating epithelial integrity and permeability. Doxycycline was administered to generate corneal epithelium-specific ablation of Klf4 in transgenic mouse. The number of epithelial cell layers and enlarged basal cells were increased in mice treated with 5 days of doxycycline, where superficial cells remained spherical [19]. After 15 days of doxycycline administration, mouse cornea revealed a defective epithelial barrier function. Tight junction proteins $\mathrm{ZO}-1$ and occludin levels in mice treated after 15 days of doxycycline were reduced compared to wild-type (WT) mice, suggesting that tight junctions are defective due to the lack of Klf4.

The integrity of epithelial basement membrane highly relies on the expression of the Klf4 gene. A decreased amount of laminin-332 expression was found in the corneal epithelial basement membrane of doxycycline-treated mice compared to that of WT mice. Yet, the expression of matrix metalloproteinase 9 (MMP9) in mice treated with doxycycline was increased, which may lead to the degradation of basement membrane. Expression of basement membrane components and epithelial markers in mice treated with doxycycline were reduced, while that of keratinised epithelia was increased. In addition, elevated cell cycle regulator Ccnd2 expression was observed, which led to the increase in epithelial layers in the cornea of Klf4 transgenic mouse. These results propose that Klf4 is important in maintaining corneal membrane integrity and homeostasis.

\section{Pax6 Gene}

Pax6 gene was widely known to be expressed in eye development [20]. Absence of this gene will lead to ocular abnormalities such as aniridia-related keratopathy [21] and limbal epithelial stem cell deficiency [22]. Epithelial cells in transgenic mice with high Pax6 level were found to be irregular and had indistinct cell junctions with enlarged microvilli [23]. In the stromal layer, keratocytes from mice with low Pax6 level had larger vacuoles, yet 
those with high Pax6 expression showed smaller ones, with both cells more highly innervated than WT keratocytes. Also, endothelial cells with low Pax6 level were bigger and slightly irregular in shape, while those with high Pax6 level were highly irregular with unclear cell borders. This indicated that under or overexpression of Pax6 gene will lead to abnormal cell morphology, affecting cell adhesion and corneal hydration.

Additionally, PAX77 ${ }^{\mathrm{Tg} /-}$ transgene, which has an overexpression of the Pax6 gene, was revealed to rescue the altered ocular phenotype in Pax6 heterozygote mice, normalizing corneal circumference and epithelial maintenance. By inducing PAX77 transgene, mosaic corneal stripe patterns were shown to be normal in heterozygotes, and putative deficiency in active limbal stem cell clones was normalised, reflecting that this transgene may be a therapy to restore corneal homeostasis.

\section{Inhibitor of Differentiation Gene}

Four Id genes (Id1-Id4) were identified as inhibitors of basic helix-loop-helix transcription factors, which contribute to Id proteins for the regulation of cell proliferation and differentiation [24]. The role of Id genes in cornea stromal homeostasis was studied using corneal fibroblast culture [25]. In the study, Id 1 and Id 2 were found in human corneal epithelial and endothelial cells. HCFs even expressed all 4 Id genes, but these genes were not found in myofibroblasts, which is a phenotypically differentiated HCF under fibrotic response.

Indeed, Id genes were discovered to be differentially regulated by TGF- $\beta 1$ and bone morphogenetic protein- 7 (BMP-7), a protein which was reported to oppose TGF- $\beta 1$ activity [26]. Fibroblasts expressed Id genes in a time-dependent manner under both TGF- $\beta 1$ and BMP-7 treatment. After stimulation by TGF- $\beta 1$ for $2-48 \mathrm{~h}$, Id $1-3$ mRNA expression peaked at the second hour but decreased at longer time points. Id 3 was especially found to have an increasing trend with TGF- $\beta 1$ treatment at 24 and $48 \mathrm{~h}$. Similarly, Id 1-3 also showed gradually increased expression with BMP-7 treatment. Moreover, under co-stimulation of TGF- $\beta 1$ and BMP-7, Id 1 was significantly increased with low expression level of $\alpha$-smooth muscle actin ( $\alpha$-SMA) in HCFs, proving the anti-fibrotic effects possessed by BMP7 in the presence of TGF- $\beta 1$. Hence, Id genes may contribute to the modulation of corneal haze by controlling fibroblast activation or their dedifferentiation.

\section{Proteins}

Pro-Fibrotic Ligands

TGF- $\beta 1$ and connective tissue growth factor (CTGF) were found to promote fibrotic response in the stromal layer of the cornea.

Transforming Growth Factor- $\beta 1$. TGF- $\beta$ performs as a cytokine in promoting cornea development and homeostasis. Activation of TGF- $\beta 1$ upon injury will disrupt the maintenance of corneal homeostasis, as previous research has shown that scar formation may be due to increased expression of TGF- $\beta 1$ [27]. The endothelial layer of TGF- $\beta$ transgenic mice at postnatal day 7 (P7) started to lose its cell-cell contact and detach from inner surface while the stroma became thicker. Concurrently, epithelial cells were found poorly differentiated. TGF- $\beta$ transgenic mice at $\mathrm{P} 14$ demonstrated a strong retention of fluorescence staining on the corneal surface by fluorescein binding, showing the surface roughness and defective epithelial layer on the cornea [14]. In addition, as TGF- $\beta 1$ is an important component in epithelial-to-mesenchymal transition (EMT), a marker for EMT, $\alpha$-SMA, was found in both corneal endothelial and epithelial layers in mice at P16, revealing that most cells have transformed into myofibroblast-like type, which lead to stromal opacification and impaired visual clarity. Endothelial cells even showed well-organised stress fibres between P21 and P30, suggesting that they may migrate as myofibroblasts into the stroma and impede normal vision. $\alpha$-SMA-expressing cells were firstly found in both endothelial and epithelial layers, subsequently in the stroma. Besides, in situ hybridisation resulted in a downregulation of $\mathrm{E}$ - and $\mathrm{N}$-cadherin in TGF- $\beta$ transgenic mice cornea, while cadherin-11 was abundant in epithelial and stroma layers, which supported that the upregulation of TGF- $\beta 1$ induces the formation of myofibroblasts in cornea. Thus, the study revealed that tight control of TGF- $\beta 1$ secretion is crucial to maintain corneal homeostasis.

Connective Tissue Growth Factor. CTGF, a protein of 349 amino acids, is a pivotal player in determining ECM remodelling and fibrosis after external disturbance [28]. CTGF immunoreactivity was shown in corneal epithelium, especially in basal layers, yet less prominent in superficial stratified layers [29]. CTGF was also found in cornea stromal keratinocytes and endothelial cells. Indeed, CTGF interacts highly with TGF- $\beta$ to induce the proliferation of telomerase-immortalised human cornea stroma fibroblast cells (THSFs) [30]. However, blocking CTGF action could hinder activation of TGF- $\beta$ in promoting THSF proliferation, which suggested that CTGF may act as a downstream mediator of TGF- $\beta$ in control- 
ling stromal fibrosis. CTGF production was further shown to be upregulated in TGF- $\beta$ stimulated cells. After blocking the c-jun $\mathrm{NH} 2$ terminal kinase (JNK) pathway with JNK1 and JNK2 siRNA, CTGF mRNA level was shown to be drastically reduced. Moreover, less PhosphoJNK $1 / 2$ was detected upon TGF- $\beta$ stimulation in THSFs pre-treated with JNK1/2 siRNA. This indicated that the JNK pathway regulates TGF- $\beta$-induced CTGF expression, leading to corneal fibrotic response upon injury. Hence, CTGF was found to promote corneal fibrosis under the influence of TGF- $\beta$.

\section{Anti-Fibrotic Ligands}

HGF and protein phosphate magnesium dependent 1A (PPM1A) were found to minimise fibrotic response in the stromal layer of the cornea.

Hepatocyte Growth Factor. Secreted by mesenchymal cells, HGF stimulates proliferation, morphogenesis and migration of most mammalian epithelial cells [31]. HGF has been proven to counteract aforementioned TGF- $\beta$ effects to achieve anti-fibrotic function in different organs such as lung [32], liver [33] and heart [34]. The addition of TGF- $\beta 1$ to HCFs for $24 \mathrm{~h}$ was shown to increase mRNA expression of $\alpha$-SMA [35], proving that TGF- $\beta 1$ induces transformation of HCFs to myofibroblasts. In addition, HGF at $5-50 \mathrm{ng} / \mathrm{mL}$ could suppress TGF- $\beta 1$ induced a-SMA expression, with $20 \mathrm{ng} / \mathrm{mL}$ HGF having the maximal inhibitory effect on keratocyte differentiation into myofibroblasts. A drastic drop in a-SMA was observed in HCFs with both TGF- $\beta 1$ and HGF added compared to the cells with TGF- $\beta 1$ only, indicating that HGF is effective in minimizing corneal stroma fibrosis.

To investigate how HGF alters the phenotype of myofibroblasts, HCFs were incubated with $5 \mathrm{ng} / \mathrm{mL}$ TGF- $\beta 1$ for $24 \mathrm{~h}$, followed by another $24-\mathrm{h}$ treatment of $20 \mathrm{ng} / \mathrm{mL}$ HGF. This resulted in an obvious drop in a-SMA mRNA level and suggested that HGF may have the potential to revert myofibroblast phenotype to fibroblast one [36]. In addition, the stiffness of TGF- $\beta 1$-treated HCF was increased. The increase in stiffness of HCFs could be greatly supressed by $20 \mathrm{ng} / \mathrm{mL}$ HGF, suggesting that the drop in rigidity may correlate with the amount of a-SMA expression since the formation of these contractile fibres was proven to contribute to increased cell stiffness [37].

Protein Phosphatase Magnesium-Dependent 1A. Belonging to the serine threonine protein phosphatase family PPM1, PPM1A regulates cellular phosphorylation, differentiation and apoptosis by various signalling pathways such as TGF- $\beta$ and p38-mitogen-activated protein kinase (MAPK) $[38,39]$. The roles of PPM1A in corneal fibrosis and neovascularisation were investigated via an ocular alkali burn model [40]. There was increased number of cells expressing high levels of a-SMA in PPM1A knockout mice, indicating scar tissue formation in the mice cornea. The stroma in these mice revealed more infiltrating inflammatory cells and proliferating cell nuclear antigen in dividing stromal cells, suggesting that PPM1A plays a role in restraining corneal inflammation. The PPM1A knockout mice also had a high expression of vascular endothelial growth factor (VEGF) in the cornea, which is associated with the formation of new blood vessels. Two days after alkali burn in PPM1 A knockout mice, transcription of TGF- $\beta$-related genes in cornea such as MMP9 and VEGF were increased.

To understand the mechanism on how PPM1A controls the TGF- $\beta$ signalling, phospho-p38 that has been indicated in the p38-MAPK pathway was investigated, as it was active in corneal keratocytes of PPM1A knockout mice even in the control group. However, after TGF- $\beta$ treatment, cell phosphorylation in keratocytes from PPM1A knockout mice was significantly increased, showing that p38 is a preferred PPM1A substrate and its dephosphorylation regulates the TGF- $\beta$ signalling cascade. MMP9 and VEGF genes were activated in PPM1A-depleted keratocytes in vitro. Blocking $\mathrm{p} 38$-MAPK inhibited TGF- $\beta$ to upregulate the expressions of MMP9 and VEGF. Thus, PPM1A was suggested to be crucial to corneal homeostasis via inhibiting the p38-MAPK pathway and interacting with TGF- $\beta$.

\section{Anti-Inflammatory Ligands}

Secreted lymphocyte antigen (Ly6)/urokinase-type plasminogen activator receptor (uPAR)-related protein-1 (SLURP1) and vasoactive intestinal peptide (VIP) were found to reduce inflammatory response in the epithelialstromal layer of the cornea.

Ly6/uPAR-Related Protein-1. SLURP1, a member of the Ly6 superfamily, is downregulated during infection to help develop protective inflammation and inhibit leukocyte infiltration [41]. Mutation of the Slurp1 gene is related to mal de Meleda [42], which contributes to transgressive keratosis and keratoderma. SLURP1 was shown to have a suppressive effect on both the proliferation and migration of human corneal limbal epithelial cells [43]. In contrast, uPAR, another Ly6 family member, was shown to play a role in regulating cell survival and invasion [44]. Its expression was upregulated during injury, and its functions were dependent on ligands including urokinase-type plasminogen activator (uPA), which was confirmed to be one of the SLUPR1-binding proteins. 
SLUPR1 was suggested to hinder the stimulatory effect of uPA on cell migration. As a result, under normal condition, SLURP1 is hypothesised to be bound with uPA and inhibit the binding of uPA to uPAR, which maintains a stable ECM without inflammation. However, under injured conditions, uPA is free to bind to UPAR since SLURP1 is downregulated for developing protective effects, hence promoting ECM degradation and inflammation. Therefore, SLUPR1 is critical to corneal homeostasis in controlling epithelial cells migration by acting as a soluble scavenger.

Vasoactive Intestinal Peptide. VIP is a neuropeptide which can be found in corneal nerves and aqueous humour of human eyes. It can inhibit pro-inflammatory cytokine production and promote healing or growth of the cornea [45]. The increase in the expression of growth factors like EGF and HGF, were shown in mice induced with Pseudomonas aeruginosa followed by VIP treatment [46]. VIP-treated cornea had increased expressions of angiogenic molecules like VEGF-A and VEGF receptor-2 (VEGFR-2), with histological examination showing greater vascularity in the peripheral cornea. All these may contribute to resistance to Pseudomonas aeruginosa keratitis. The increase in EGF, fibroblast growth factor (FGF), HGF and VEGF-A was further confirmed at 5 days post-infection in the epithelium and stroma of VIP-added cornea.

Antimicrobial $\beta$-defensins were also detected in infected cornea. After treatment with VIP, the amount of mouse $\beta$-defensins 2 was increased in corneal epithelium and stroma at 5 days post-infection. In addition, the VIPtreated samples had decreased bacterial counts at 1,3 and 5 days post-infection. Hence, this study supported the idea that VIP is involved in immune homeostasis and regulates other growth factors, thus facilitating corneal wound healing after keratitis.

Wound-Repairing Ligands

Lumican and MMP12 were found to play a role in promoting wound healing in the cornea.

Lumican. Lumican, one of the proteoglycans in the stroma, is expressed by mesenchymal fibroblasts. It binds to collagens and regulates collagen fibril growth [47]. Lumican-deficient mice were found to lose corneal transparency, and this disorder was associated with abnormal collagen fibril architecture [48]. Among 2,173 proteins identified via iTRAQ labelling, 113 corneal proteins were upregulated, while 47 were downregulated in both lumican-expressing and lumican-deficient mice [49]. Basement membrane proteins including laminin-beta 2 and perlecan were found to be elevated in lumican-deficient

Cornea Epithelial-Stromal Homeostasis mice while small leucine-rich repeat proteoglycans such as decorin and biglycan were decreased. Collagen type II, which is normally absent in mammalian cornea, was detected with an increased amount in lumican-deficient mice. Collagen type IV, XII, XIV were also increased slightly; yet, the level of cytokeratin 8 dropped in the same study. In addition, the expressions of dermatopontin, insulin-like growth factor-binding protein 2 and fibromodulin were increased, but at the same time, expressions of keratin 8 , biglycan and keratocan were decreased. From the above protein changes, lumican is important in regulating ECM and cellular protein levels, thus disrupted corneal integrity and various ocular disorders such as keratoconus occur upon reduced production.

Matrix Metalloproteinase 12. MMP, a family of ECM proteinase, has been found to be a regulator in repairing process and remodelling. MMP12 was suggested to have protective effects on corneal fibrosis and maintaining stromal transparency by controlling immune cells [50]. After removal of the epithelial layer in mouse cornea, the MMP12 mRNA expression was increased at $2 \mathrm{~h}$ post-injury with a highest level observed at $8 \mathrm{~h}$ [51]. Yet, MMP12 expression declined gradually after 4 days of injury, showing that MMP12 was expressed immediately after injury. MMP12 knockout mice had significant delay in epithelial closure at 8 and $18 \mathrm{~h}$ after injury when compared to WT mice, revealing that MMP12 has a role in epithelial repair.

A slower migration rate in scratch wound assay was shown in the epithelial cells of MMP12 knockout mice when compared to cells from WT mice in vitro. Only $25 \%$ of the wound recovered in MMP12 knockout epithelial cells, yet WT mice cells had a $37 \%$ of recovered wound, which further proved that MMP12 is effective in promoting healing of the epithelial wound. Moreover, MMP12 knockout mice were found to have a drop in neutrophil infiltration in the anterior stroma when compared to WT mice, demonstrating that MMP12 positively recruits neutrophils upon epithelial injury to combat against infection.

\section{Growth Factors}

Platelet-derived growth factor-BB isoform (PDGF$\mathrm{BB}$ ) and basic FGF (bFGF) promote corneal fibroblast migration and ECM synthesis during stromal repair.

$P D G F-B B$ and $b F G F$. Previous studies showed that PDGF-BB and bFGF are involved in corneal wound healing, but their actions on ECM synthesis were not well understood $[52,53]$. After scratch wound injury, PDGF-BB and bFGF-treated HCF wound had a similar healing pattern, and the wounds were completely closed at day 10 , 
while wound in serum-free media had a slower healing rate [54]. In the same study, PDGF-BB-treated cells also had a higher level of collagen III expression, which is a marker of repair matrix synthesis. Integrin subunits $\alpha_{5}$ and $\beta_{1}$ were highly expressed in PDGF-BB-treated HCFs but not in bFGF-treated cells. The proteoglycan SDC4 had an increased mRNA expression in HCFs added with PDGF-BB and bFGF, suggesting that these growth factors upregulate new ECM synthesis. Unlike a homogeneous cytoplasmic distribution in normal situation, SDC4 was in the trailing edge of HCFs that were pre-treated with PDGF-BB. In addition, perlecan mRNA expression was increased in PDGF-BB and bFGF-treated cells upon injury, suggesting that these growth factors promote the secretion of various proteins which may facilitate corneal repair. These proteoglycans are markers of non-fibrotic repair phenotype and are vital in maintaining corneal homeostasis.

\section{Receptors}

VEGFR-1, aquaporin-5 (AQP5) and transient receptor potential vanilloid subtype 1 (TRPV1) were found to be involved in maintaining the epithelial-stromal homeostasis.

Vascular Endothelial Growth Factor Receptor-1. In light of the involvement of VEGF in maintaining cornea avascularity, the role of VEGFR in regulating stromal fibroblast network and corneal integrity was investigated. A circuit of a communication network between fibroblasts has been found in the stroma, which is crucial to maintain corneal transparency [55]. To investigate the role of VEGFR-1 in forming intercellular networks, HCFs that did not form networks were studied. Among them, HCFs with a high level of VEGFR-1 showed an increased number of cell interconnections yet HCFs with a low level of VEGFR-1 did not show obvious response upon addition of exogenous VEGF. Also, inducing HCFs with high VEGFR-1 expression with VEGF-A and VEGF-C led to more cell migration. Nonetheless, addition of Avastin, an anti-VEGF agent, showed a negative result in cell motility, proving that VEGF controls fibroblast organisation and migration. Hence, a well-regulated VEGFR-1 expression in fibroblast influences cell arrangement and thus visual clarity.

When fibroblasts were transfected with VEGFR-1 siRNA, the cell motility and network formation drastically decreased. However, transfection with VEGFR-2 and 3 siRNA did not show significant influence on network formation. Eventually, VEGFR-1 resulted in a decrease with increasing age, suggesting the loss of such a receptor leads to disorganised stromal network and hence poor vision in elderly.

Aquaporin-5. AQP water channels, responsible for water conductance across the plasma membrane, are highly found in lens to maintain homeostasis [56]. AQP5 transcript expression and AQP5 protein were both found in mouse cornea and lens [57]. AQP5 expression was found to be low in keratocytes close to Bowman's membrane, while corneal basal columnar epithelium had the highest abundance of AQP5. The AQP5 in the stromal layer was suggested to maintain corneal hydration since epithelial water permeability was reduced and corneal thickness was increased in AQP5 knockout mice. Moreover, the recovery rate of the epithelial cells of AQP5 knockout mice was significantly decreased after putting in a hypotonic condition when compared to the cells of WT mice. AQP5 was demonstrated to be present in the cornea epithelium, and the loss of AQP5 may lead to dry eye disease and corneal oedema [58].

Cyclic adenosine monophosphate (cAMP) was observed to regulate epithelial cells' AQP5 expression through the phosphokinase A (PKA) pathway. In vitro studies showed that when Madin-Darby canine kidney cells, which express AQP5, were exposed to a PKA agonist, mp-cAMP, the AQP5 plasma membrane localisation dropped substantially. However, when a PKA antagonist, $\mathrm{H}-89$, was added, the AQP5 membrane localisation and abundance showed an obvious increase. This reflected that PKA-mediated regulation of AQP5s may be a therapeutic target for corneal disease.

Transient Receptor Potential Vanilloid Subtype 1. As a non-selective cation channel, TRPV1 was found to mediate inflammatory responses by releasing endogenous agonists and metabolites [59]. The role of TRPV1 in inhibiting corneal transparency upon wound healing was studied [60]. Reverse transcriptase-dependent generation of products for TRPV1 was found in HCFs and HCECs along membrane perimeter and perinuclear regions. Addition of capsaicin, a selective TRPV1 agonist, resulted in a 2 -fold $\mathrm{Ca}^{2+}$ concentration rise in HCFs and such an increase was blocked by adding capsazepine, a TRPV1 antagonist, showing that TRPV1 expression was functional. Moreover, induction of capsaicin on TRPV1 led to a rise in $\mathrm{Ca}^{2+}$, resulting in MAPK phosphorylation in both HCECs and HCFs. Capsaicin-induced terminal kinase phosphorylation was found absent with capsazepine on TRPV1. In cells transduced with TRPV1 siRNA, capsaicin-induced MAPK activation was diminished, showing that TRPV1 was involved in eliciting the responses. Via MAPK stimulation, activation of TRPV1 was observed to 
increase IL-6 expression in HCFs, which leads to corneal fibrosis [61]. Presence of a p38 MAPK inhibitor resulted in an inhibition of IL- 6 rise, illustrating that TRPV1-mediated IL- 6 can be elicited by p38 MAPK stimulation. Hence, suppression of TRPV 1 activation may pave a way to the restoration of corneal homeostasis [62].

\section{Lipid}

C6 ceramide was found to be a lipid involved in the epithelial-stromal layer of the cornea.

\section{C6 Ceramide}

Cell death is considered an essential step in maintaining corneal homeostasis. Ceramide, a second messenger in regulating cell proliferation, differentiation and growth, was proven to play a significant role in stromal cell death after epithelial injury [63]. A study showed that a larger dose of short-chain ceramide causes a greater proportion of cell death of HCF and a drastic reduction in cell viability [64]. Ceramide was further revealed to cause mitochondrial dysfunction of HCF with an increased amount of cytochrome $\mathrm{C}$ released from mitochondria. Exposure of HCFs to ceramide also resulted in enhanced production of mitochondrial reactive oxygen species and a lowered mitochondrial membrane potential.

Increased JNK phosphorylation was observed in ceramide-treated HCF samples, suggesting that ceramide may regulate harikari gene (HRK) expression, which has been found to initiate cell death under physiological or pathological circumstances. HCFs with ceramide treatment had a prominent upregulation of HRK expression, which initiated cell death under physiological condition. An association between HRK and mitochondrial protein p32 was established by co-immunoprecipitation, reflecting that HRK translocated to mitochondria and interacted with p32, which most likely contributed to cell death. Thus, the results suggested that in corneal homeostasis, cell death of HCFs after ceramide secretion is due to mitochondrial dysfunction mediated by HRK expression [64-66].

\section{Discussion}

Cornea stromal scarring is the fourth common cause of worldwide blindness, which contributes to $5.1 \%$ of the cases according the World Health Organisation [67]. Patients with corneal opacity generally result in a lowered vision-related quality of life and experience social problems such as fearing to lose residual vision and going out at night [68]. In spite of the current treatments on corneal disorders with medical advancement, the consequences of corneal injury such as opacification and chronic inflammation remain poorly treated [69]. To identify therapeutic targets for corneal scarring, the understanding of the response of cornea to external disturbance and therefore the cornea epithelial-stromal homeostasis is of utmost importance. Previous aforementioned studies suggested that a particular gene or cell type involved in corneal homeostasis can be targeted in the course of developing novel approaches to treat stromal scarring. Hence, an in-depth understanding of the molecular pathways enables the development of new targeted therapies to minimise scarring or prolonged inflammation.

To tackle the problem of stromal fibrosis, proposed effective managements have targeted TGF- $\beta 1$ because studies have shown the upregulation of TGF- $\beta 1$ could result in differentiation of keratocytes to myofibroblasts [4]. Many pharmaceutics block the action of TGF- $\beta 1$ secreted from the epithelium to improve corneal clarity [14]. Nonetheless, blockage of other relevant molecules could also be considered to minimise fibrosis. For example, CTGF production was found to be mediated by the JNK pathway; thus, inhibitors of the JNK pathway may be a means to hinder CTGF action [70]. Similarly, TRPV1 can be blocked by specific inhibitors such as capsazepine to reduce dysregulated ECM deposition. In contrast, proteins that inhibit excessive fibrosis may promote wound healing outcome. For example, HGF can suppress TGF-promoted myofibroblast phenotype [71], while PPM1A can terminate TGF response and reduce angiogenesis [38]. Lumican can maintain a proper collagen fibril architecture, contributing to transparency and stability [49]. These lines of evidence may suggest that proteins can be administered to the cornea exogenously depending on the specific aim to be achieved, thus promoting a better prognosis of the healing process with minimal scarring. Furthermore, understanding the roles played by each protein favours a more specific healing outcome. For instance, to elicit protective inflammation and epithelial cell migration, MMP12 should be upregulated, while PPM1A action ought to be suppressed since their mechanisms of action are antagonistic [40,51]. Tear drops or ointment can be formulated in clinical settings based on the findings in in vitro and in vivo studies. For instance, recombinant nerve growth factor, which was found to accelerate cornea healing in both human cell culturing and rat in vivo research, has been translated to clinical patients in dry eye disease $[72,73]$. It was tested to be safe and effective in improving signs and symptoms in patients at different dosage. Hence, laboratory understanding of corneal pathophysiology 


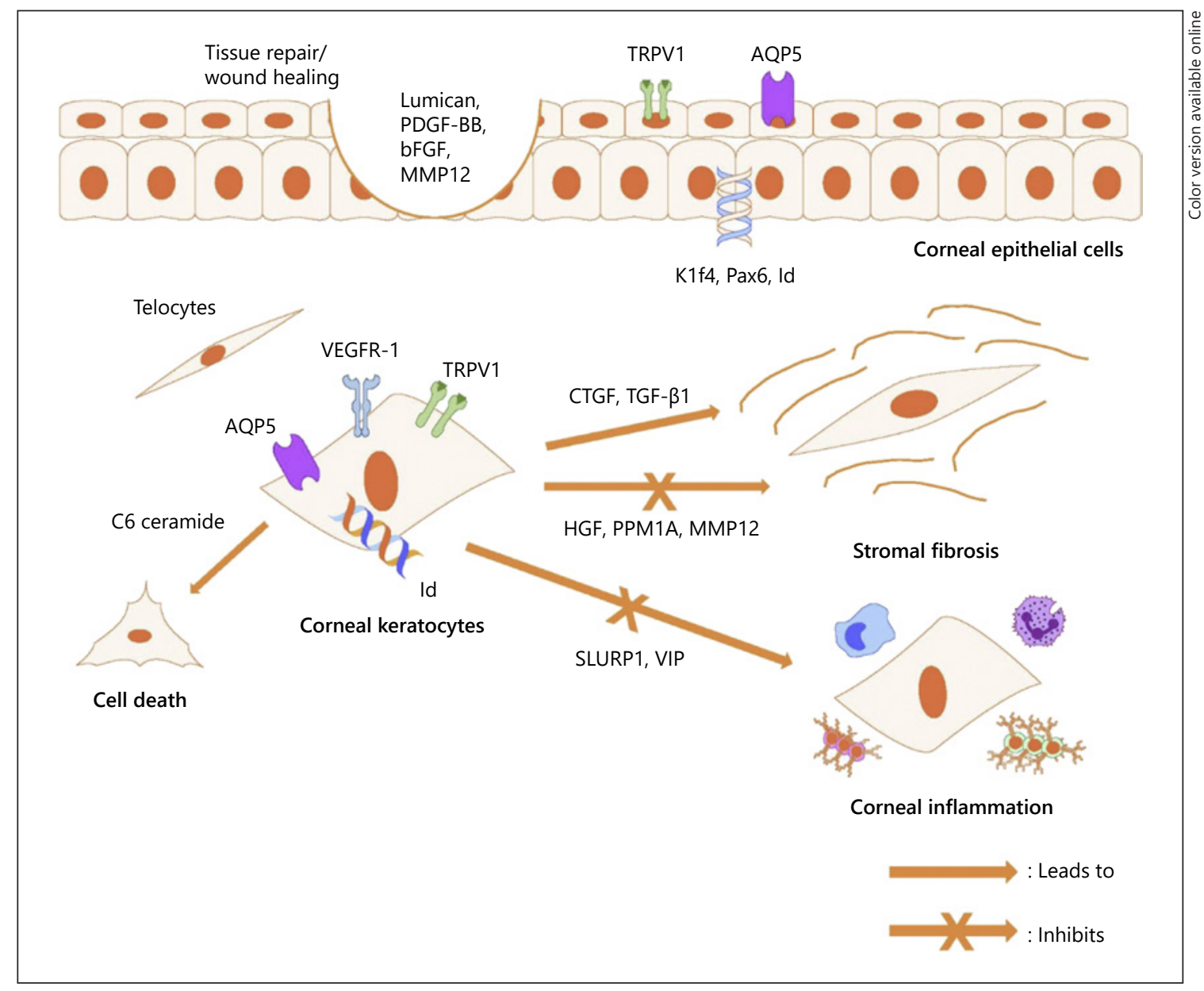

Fig. 2. Schematic diagram of cells, genes, proteins and lipid involved in the cornea epithelial-stromal homeostasis.

may prompt the use of novel topical drops, suspensions or solutions in clinical settings.

Different genes are being identified to be responsible for maintaining epithelial integrity and homeostasis. The Klf4 gene influences the differentiation and barrier functions of the epithelial cells, which prevents Meesmann dystrophy and dry eye diseases [19]. Pax6 heterozygotes result in ARK and LESC deficiency, and it was later discovered that the addition of transgene PAX77 helps compensate such corneal defects [23]. These genes are essential in formulating treatments such as gene therapy for congenital ocular diseases, particularly in infants who show epithelial fragility, and such understanding of the related genetic pathophysiology may bring up novel molecular treatments for these diseases. For instance, in the treatment of Leber congenital amaurosis, RPE65 gene therapy could be included in phase 1 human clinical trials if it proves successful in experimental models [74]. Simi- larly, with the discovery of the contribution of embryonic stem cells, adult stem cells and induced pluripotent stem cells to differentiating into CECs, these stem cells have been adopted as candidates for regenerative medicine and curation of ocular surface diseases [75]. Therefore, given the superficial and easily accessible location of the cornea, gene and cell therapy could be ways to treat corneal disorders.

Most clinicians usually prescribe topical corticosteroids which downregulate TGF- $\beta 1$ to minimise corneal scarring after injury or surgery despite their significant side effects [76]. To solve this problem, in the long-term, novel topical drugs, gene or cell therapies can be developed by targeting different proteins, genes or cells that can reduce fibrosis or repair epithelial damage. A particular study highlighted that HGF was shown to have the potential ability to reverse the differentiation of human myofibroblasts back into keratocytes [35], providing 
hope for patients suffering from scarring to regain visual clarity through pharmaceutical means. The effects of other aforementioned proteins on the reversed differentiation of myofibroblasts could also be investigated, and further studies on post-treatment of keratocytes after injury are warranted. Nonetheless, one of the limitations of the current research on this area is that the relevant evidence remains on the laboratory scale, where clinical trials are required. Also, the effects of exogenous administration of a molecule to other cell types apart from cornea such as conjunctival epithelium and goblet cells were not investigated, which may potentially disrupt the homeostasis of other neighbouring tissues of the cornea such as conjunctiva, lacrimal glands and sclera. Another limitation would be the lack of further in vitro or in vivo studies to prove that administration of exogenous molecules, which were found to play an important role in the cornea physiology, can improve the healing outcome. This suggests that in the future randomised controlled clinical trials with human subjects can be conducted when in vitro and in vivo studies showed repeated positive results such as those with MMP12 and PPM1A. With more comprehensive knowledge regarding corneal homeostasis, improved and specific clinical outcomes can be achieved with fewer adverse effects using targeted pharmaceutical agents.

\section{Conclusion}

To promote a healthy cornea epithelium and stroma, the dynamics of various cells, genes, proteins and lipid ought to be tightly regulated and maintained in a stable manner (Fig. 2), as they help to maintain epithelial integ- rity, reduce corneal fibrosis and promote protective inflammation. A thorough understanding of the roles of each relevant molecule will be essential to facilitate the development of therapeutic strategies for minimizing specific corneal conditions, such as stromal scarring and disrupted epithelial basement membrane. Hence, we believe that the investigations of the molecular pathways highlighted in this review will hint at more future therapeutic tools to treat corneal diseases.

\section{Statement of Ethics}

Ethics approval is not required since there is no involvement of human and animals.

\section{Conflict of Interest Statement}

The authors have no conflicts of interest to declare.

\section{Funding Sources}

No funding or grant support.

\section{Author Contributions}

All authors attest that they meet the current ICMJE criteria for authorship. H.L.W. and K.C.S. were involved in study design, data collection, data analysis, manuscript writing and editing. S.H.L.P., Y.B., A.C.Y.L., V.J. and Y.K.C. were involved in data collection, data analysis, manuscript writing and editing. All authors have approved the final version of the manuscript.

\section{References}

1 Lu L, Reinach PS, Kao WW. Corneal epithelial wound healing. Exp Biol Med (Maywood). $2001 \mathrm{Jul} ; 226(7): 653-64$.

2 Mastropasqua L, Massaro-Giordano G, Nubile M, Sacchetti M. Understanding the Pathogenesis of Neurotrophic Keratitis: The Role of Corneal Nerves. J Cell Physiol. 2017 Apr;232(4):717-24.

3 Dua HS, Said DG, Messmer EM, Rolando M, Benitez-Del-Castillo JM, Hossain PN, et al. Neurotrophic keratopathy. Prog Retin Eye Res. 2018 Sep;66:107-31.

4 Medeiros CS, Marino GK, Santhiago MR, Wilson SE. The Corneal Basement Membranes and Stromal Fibrosis. Invest Ophthalmol Vis Sci. 2018 Aug;59(10):4044-53.
5 Meek KM, Leonard DW, Connon CJ, Dennis S, Khan S. Transparency, swelling and scarring in the corneal stroma. Eye (Lond). 2003 Nov;17(8):927-36.

6 Ouyang H, Xue Y, Lin Y, Zhang X, Xi L, Patel $\mathrm{S}$, et al. WNT7A and PAX6 define corneal epithelium homeostasis and pathogenesis. $\mathrm{Na}-$ ture. 2014 Jul;511(7509):358-61.

7 Frank MH, Frank NY. Restoring the cornea from limbal stem cells. Regen Med. 2015; 10(1):1-4.

8 Reins RY, Courson J, Lema C, Redfern RL. MyD88 contribution to ocular surface homeostasis. PLoS One. 2017 Aug;12(8):e0182153.

9 Suvas S. Role of Substance P Neuropeptide in Inflammation, Wound Healing, and Tissue Homeostasis. J Immunol. 2017 Sep;199(5): 1543-52.
10 Wilson SE, Liu JJ, Mohan RR. Stromal-epithelial interactions in the cornea. Prog Retin Eye Res. 1999 May;18(3):293-309.

11 Gabison EE, Huet E, Baudouin C, Menashi S. Direct epithelial-stromal interaction in corneal wound healing: role of EMMPRIN/ CD147 in MMPs induction and beyond. Prog Retin Eye Res. 2009 Jan;28(1):19-33.

12 Kobayashi T, Shiraishi A, Hara Y, Kadota Y, Yang L, Inoue T, et al. Stromal-epithelial interaction study: the effect of corneal epithelial cells on growth factor expression in stromal cells using organotypic culture model. Exp Eye Res. 2015 Jun;135:109-17. 
13 McClintock JL, Ceresa BP. Transforming growth factor-\{alpha\} enhances corneal epithelial cell migration by promoting EGFR recycling. Invest Ophthalmol Vis Sci. 2010 Jul; 51(7):3455-61.

14 Reneker LW, Bloch A, Xie L, Overbeek PA, Ash JD. Induction of corneal myofibroblasts by lens-derived transforming growth factor betal (TGFbeta1): a transgenic mouse model. Brain Res Bull. 2010 Feb;81(2-3):287-96.

15 Chang Y, Li C, Gan L, Li H, Guo Z. Telocytes in the Spleen. PLoS One. 2015 Sep; 10(9):e0138851.

16 Loyer X, Zlatanova I, Devue C, Yin M, Howangyin KY, Klaihmon P, et al. Intra-Cardiac Release of Extracellular Vesicles Shapes Inflammation Following Myocardial Infarction. Circ Res. 2018 Jun;123(1):100-6.

17 Torricelli AA, Singh V, Santhiago MR, Wilson SE. The corneal epithelial basement membrane: structure, function, and disease. Invest Ophthalmol Vis Sci. 2013 Sep;54(9): 6390-400.

18 Swamynathan SK, Katz JP, Kaestner KH, Ashery-Padan R, Crawford MA, Piatigorsky J. Conditional deletion of the mouse Klf4 gene results in corneal epithelial fragility, stromal edema, and loss of conjunctival goblet cells. Mol Cell Biol. 2007 Jan;27(1):182-94.

19 Delp EE, Swamynathan S, Kao WW, Swamynathan SK. Spatiotemporally Regulated Ablation of Klf4 in Adult Mouse Corneal Epithelial Cells Results in Altered Epithelial Cell Identity and Disrupted Homeostasis. Invest Ophthalmol Vis Sci. 2015 Jun;56(6):3549-58.

20 Remez LA, Onishi A, Menuchin-Lasowski Y, Biran A, Blackshaw S, Wahlin KJ, et al. Pax6 is essential for the generation of late-born retinal neurons and for inhibition of photoreceptor-fate during late stages of retinogenesis. Dev Biol. 2017 Dec;432(1):140-50.

21 Zhang R, Linpeng S, Wei X, Li H, Huang Y, Guo J, et al. Novel variants in PAX6 gene caused congenital aniridia in two Chinese families. Eye (Lond). 2017 Jun;31(6):956-61.

22 Laggner M, Pollreisz A, Schmidinger G, Schmidt-Erfurth U, Chen YT. Autophagy mediates cell cycle response by regulating nucleocytoplasmic transport of PAX6 in limbal stem cells under ultraviolet-A stress. PLoS One. 2017 Jul;12(7):e0180868.

23 Mort RL, Bentley AJ, Martin FL, Collinson JM, Douvaras P, Hill RE, et al. Effects of aberrant Pax6 gene dosage on mouse corneal pathophysiology and corneal epithelial homeostasis. PLoS One. 2011;6(12):e28895.

24 Norton JD. ID helix-loop-helix proteins in cell growth, differentiation and tumorigenesis. J Cell Sci. 2000 Nov; 113(Pt 22):3897-905.

25 Mohan RR, Morgan BR, Anumanthan G, Sharma A, Chaurasia SS, Rieger FG. Characterization of Inhibitor of differentiation (Id) proteins in human cornea. Exp Eye Res. 2016 May;146:145-53.
26 Tandon A, Sharma A, Rodier JT, Klibanov AM, Rieger FG, Mohan RR. BMP7 gene transfer via gold nanoparticles into stroma inhibits corneal fibrosis in vivo. PLoS One. 2013 Jun;8(6):e66434.

27 Jester JV, Barry-Lane PA, Petroll WM, Olsen DR, Cavanagh HD. Inhibition of corneal fibrosis by topical application of blocking antibodies to TGF beta in the rabbit. Cornea. 1997 Mar;16(2):177-87.

28 Gibson DJ, Pi L, Sriram S, Mao C, Petersen BE, Scott EW, et al. Conditional knockout of CTGF affects corneal wound healing. Invest Ophthalmol Vis Sci. 2014 Apr;55(4):2062-70.

29 van Setten GB, Trost A, Schrödl F, KaserEichberger A, Bogner B, van Setten M, et al. Immunohistochemical Detection of CTGF in the Human Eye. Curr Eye Res. 2016 Dec; 41(12):1571-9.

30 Chang Y, Wu XY. JNK1/2 siRNA inhibits transforming-growth factor-beta1-induced connective tissue growth factor expression and fibrotic function in THSFs. Mol Cell Biochem. 2010 Feb;335(1-2):83-9.

31 Imamura R, Matsumoto K. Hepatocyte growth factor in physiology and infectious diseases. Cytokine. 2017 Oct;98:97-106.

32 Nita I, Hostettler K, Tamo L, Medová M, Bombaci G, Zhong J, et al. Hepatocyte growth factor secreted by bone marrow stem cell reduce ER stress and improves repair in alveolar epithelial II cells. Sci Rep. 2017 Feb;7(1): 41901.

33 Abd-Elgawad H, Abu-Elsaad N, El-Karef A, Ibrahim T. Piceatannol increases the expression of hepatocyte growth factor and IL-10 thereby protecting hepatocytes in thioacetamide-induced liver fibrosis. Can J Physiol Pharmacol. 2016 Jul;94(7):779-87.

34 Yi X, Li X, Zhou Y, Ren S, Wan W, Feng G, et al. Hepatocyte growth factor regulates the TGF- $\beta 1$-induced proliferation, differentiation and secretory function of cardiac fibroblasts. Int J Mol Med. 2014 Aug;34(2):381-90.

35 Miyagi H, Jalilian I, Murphy CJ, Thomasy SM. Modulation of human corneal stromal cell differentiation by hepatocyte growth factor and substratum compliance. Exp Eye Res. 2018 Nov; 176:235-42.

$36 \mathrm{Bu}$ SC, Kuijer R, van der Worp RJ, van Putten SM, Wouters O, Li XR, et al. Substrate Elastic Modulus Regulates the Morphology, Focal Adhesions, and a-Smooth Muscle Actin Expression of Retinal Müller Cells. Invest Ophthalmol Vis Sci. 2015 Sep;56(10):5974-82.

37 Yan T, Sun R, Deng H, Tan B, Ao N. The morphological and biomechanical changes of keratocytes cultured on modified $p$ (HEMAMMA) hydrogel studied by AFM. Scanning. 2009 Nov-Dec;31(6):246-52.

38 Shohat M, Ben-Meir D, Lavi S. Protein phosphatase magnesium dependent 1A (PPM1A) plays a role in the differentiation and survival processes of nerve cells. PLoS One. 2012; 7(2):e32438.
39 Lin X, Duan X, Liang YY, Su Y, Wrighton KH, Long J, et al. PPM1A Functions as a Smad Phosphatase to Terminate TGF $\beta$ Signaling. Cell. 2016 Sep;166(6):1597.

40 Dvashi Z, Sar Shalom H, Shohat M, Ben-Meir D, Ferber S, Satchi-Fainaro R, et al. Protein phosphatase magnesium dependent $1 \mathrm{~A}$ governs the wound healing-inflammation-angiogenesis cross talk on injury. Am J Pathol. 2014 Nov;184(11):2936-50.

41 Swamynathan S, Buela KA, Kinchington P, Lathrop KL, Misawa H, Hendricks RL, et al. Klf4 regulates the expression of Slurp1, which functions as an immunomodulatory peptide in the mouse cornea. Invest Ophthalmol Vis Sci. 2012 Dec;53(13):8433-46.

42 Radiono S, Pramono ZA, Oh GG, Surana U, Widiyani S, Danarti R. Identification of novel homozygous SLURP1 mutation in a Javanese family with Mal de Meleda. Int J Dermatol. 2017 Nov;56(11):1161-8.

43 Swamynathan S, Swamynathan SK. SLURP-1 modulates corneal homeostasis by serving as a soluble scavenger of urokinase-type plasminogen activator. Invest Ophthalmol Vis Sci. 2014 Aug;55(10):6251-61.

44 Loughner CL, Bruford EA, McAndrews MS, Delp EE, Swamynathan S, Swamynathan SK. Organization, evolution and functions of the human and mouse Ly6/uPAR family genes. Hum Genomics. 2016 Apr;10(1):10.

45 Maugeri G, D’Amico AG, Gagliano C, Saccone S, Federico C, Cavallaro S, et al. VIP Family Members Prevent Outer Blood Retinal Barrier Damage in a Model of Diabetic Macular Edema. J Cell Physiol. 2017 May;232(5): 1079-85.

46 Jiang X, McClellan SA, Barrett RP, Berger EA, Zhang Y, Hazlett LD. VIP and growth factors in the infected cornea. Invest Ophthalmol Vis Sci. 2011 Aug;52(9):6154-61.

47 Rada JA, Cornuet PK, Hassell JR. Regulation of corneal collagen fibrillogenesis in vitro by corneal proteoglycan (lumican and decorin) core proteins. Exp Eye Res. 1993 Jun;56(6): 635-48.

48 Chakravarti S, Petroll WM, Hassell JR, Jester JV, Lass JH, Paul J, et al. Corneal opacity in lumican-null mice: defects in collagen fibril structure and packing in the posterior stroma. Invest Ophthalmol Vis Sci. 2000 Oct;41(11): 3365-73.

49 Shao H, Chaerkady R, Chen S, Pinto SM, Sharma R, Delanghe B, et al. Proteome profiling of wild type and lumican-deficient mouse corneas. J Proteomics. 2011 Sep;74(10):1895905.

50 Chan MF, Li J, Bertrand A, Casbon AJ, Lin JH, Maltseva I, et al. Protective effects of matrix metalloproteinase-12 following corneal injury. J Cell Sci. 2013 Sep;126(Pt 17):3948-60.

51 Wolf M, Maltseva I, Clay SM, Pan P, Gajjala A, Chan MF. Effects of MMP12 on cell motility and inflammation during corneal epithelial repair. Exp Eye Res. 2017 Jul;160:11-20. 
52 Imanishi J, Kamiyama K, Iguchi I, Kita M, Sotozono C, Kinoshita S. Growth factors: importance in wound healing and maintenance of transparency of the cornea. Prog Retin Eye Res. 2000 Jan;19(1):113-29.

53 Etheredge L, Kane BP, Hassell JR. The effect of growth factor signaling on keratocytes in vitro and its relationship to the phases of stromal wound repair. Invest Ophthalmol Vis Sci. 2009 Jul;50(7):3128-36.

54 Gallego-Muñoz P, Ibares-Frías L, Garrote JA, Valsero-Blanco MC, Cantalapiedra-Rodríguez R, Merayo-Lloves J, et al. Human corneal fibroblast migration and extracellular matrix synthesis during stromal repair: role played by platelet-derived growth factor- $\mathrm{BB}$, basic fibroblast growth factor, and transforming growth factor- $\beta 1$. J Tissue Eng Regen Med. 2018 Feb;12(2):e737-46.

55 Kang GM, Ko MK. Morphological characteristics and intercellular connections of corneal keratocytes. Korean J Ophthalmol. 2005 Sep; 19(3):213-8.

56 Schey KL, Petrova RS, Gletten RB, Donaldson PJ. The Role of Aquaporins in Ocular Lens Homeostasis. Int J Mol Sci. 2017 Dec; 18(12):E2693

57 Kumari SS, Varadaraj M, Yerramilli VS, Menon AG, Varadaraj K. Spatial expression of aquaporin 5 in mammalian cornea and lens, and regulation of its localization by phosphokinase A. Mol Vis. 2012;18:957-67.

58 Verkman AS, Ruiz-Ederra J, Levin $\mathrm{MH}$. Functions of aquaporins in the eye. Prog Retin Eye Res. 2008 Jul;27(4):420-33.

59 Bertin S, de Jong PR, Jefferies WA, Raz E. Novel immune function for the TRPV1 channel in T lymphocytes. Channels (Austin). 2014;8(6):479-80.

60 Yang Y, Yang H, Wang Z, Mergler S, Wolosin JM, Reinach PS. Functional TRPV1 expression in human corneal fibroblasts. Exp Eye Res. 2013 Feb;107:121-9.
61 Becker J, Salla S, Dohmen U, Redbrake C, Reim M. Explorative study of interleukin levels in the human cornea. Graefes Arch Clin Exp Ophthalmol. 1995 Dec;233(12):766-71.

62 Modell H, Cliff W, Michael J, McFarland J, Wenderoth MP, Wright A. A physiologist's view of homeostasis. Adv Physiol Educ. 2015 Dec;39(4):259-66.

63 Castro BM, Prieto M, Silva LC. Ceramide: a simple sphingolipid with unique biophysical properties. Prog Lipid Res. 2014 Apr;54:5367.

64 Rizvi F, Heimann T, Herrnreiter A, O’Brien WJ. Mitochondrial dysfunction links ceramide activated HRK expression and cell death. PLoS One. 2011 Mar;6(3):e18137.

65 Gurzov EN, Ortis F, Cunha DA, Gosset G, Li $M$, Cardozo AK, et al. Signaling by IL1 beta+IFN-gamma and ER stress converge on DP5/Hrk activation: a novel mechanism for pancreatic beta-cell apoptosis. Cell Death Differ. 2009 Nov; 16(11):1539-50.

66 Naegele J, Lombroso PJ. Development of the cerebral cortex: VIII. Apoptosis: neuronal Hari-Kari. J Am Acad Child Adolesc Psychiatry. 1998 Aug;37(8):890-2.

67 Whitcher JP, Srinivasan M, Upadhyay MP. Corneal blindness: a global perspective. Bull World Health Organ. 2001;79(3):214-21.

68 Vashist P, Gupta N, Tandon R, Gupta SK, Dwivedi S, Mani K. Population-based assessment of vision-related quality of life in corneal disease: results from the CORE study. $\mathrm{Br}$ J Ophthalmol. 2016 May;100(5):588-93.

69 Moore BA, Teixeira LB, Sponsel WE, Dubielzig RR. The consequences of avian ocular trauma: histopathological evidence and implications of acute and chronic disease. Vet Ophthalmol. 2017 Nov;20(6):496-504.

70 Blalock TD, Duncan MR, Varela JC, Goldstein MH, Tuli SS, Grotendorst GR, et al. Connective tissue growth factor expression and action in human corneal fibroblast cultures and rat corneas after photorefractive keratectomy. Invest Ophthalmol Vis Sci. 2003 May;44(5):1879-87.
71 Miyagi H, Thomasy SM, Russell P, Murphy CJ. The role of hepatocyte growth factor in corneal wound healing. Exp Eye Res. 2018 Jan;166:49-55.

72 Lambiase A, Manni L, Bonini S, Rama P, Micera A, Aloe L. Nerve growth factor promotes corneal healing: structural, biochemi$\mathrm{cal}$, and molecular analyses of rat and human corneas. Invest Ophthalmol Vis Sci. 2000 Apr; 41(5):1063-9.

73 Sacchetti M, Lambiase A, Schmidl D, Schmetterer L, Ferrari M, Mantelli F, et al. Effect of recombinant human nerve growth factor eye drops in patients with dry eye: a phase IIa, open label, multiple-dose study. Br J Ophthalmol. 2020 Jan;104(1):127-35.

74 Liu MM, Tuo J, Chan CC. Gene therapy for ocular diseases. Br J Ophthalmol. 2011 May; 95(5):604-12.

75 Hsu CC, Peng CH, Hung KH, Lee YY, Lin TC, Jang SF, et al. Stem Cell Therapy for Corneal Regeneration Medicine and Contemporary Nanomedicine for Corneal Disorders. Cell Transplant. 2015;24(10):1915-30.

76 Kwok SS, Shih KC, Bu Y, Lo AC, Chan TC, Lai JS, et al. Systematic Review on Therapeutic Strategies to Minimize Corneal Stromal Scarring After Injury. Eye Contact Lens. 2019 Nov;45(6):347-55.

77 Marini M, Mencucci R, Rosa I, Favuzza E, Guasti D, Ibba-Manneschi L, et al. Telocytes in normal and keratoconic human cornea: an immunohistochemical and transmission electron microscopy study. J Cell Mol Med. 2017 Dec;21(12):3602-3611.

78 Berthaut A, Mirshahi P, Benabbou N, Azzazene D, Bordu C, Therwath A, et al. Vascular endothelial growth factor receptor-1 (VEGFR-1) expression in human corneal fibroblast decreased with age. Mol Vis. 2009 Sep 29;15: 1997-2007. 